\title{
A grey relational analysis based study on green degree evaluation of urban logistics
}

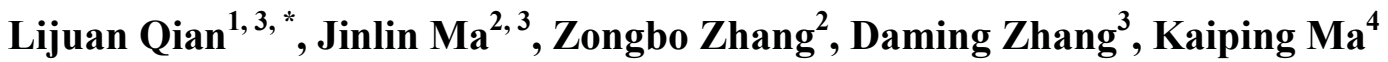 \\ ${ }^{1}$ The Faculty of Science, Jiangsu University, Zhenjiang, Jiangsu, 212013, P. R. China \\ ${ }^{2}$ School of Automobile and Traffic Engineering, Jiangsu University, Zhenjiang, Jiangsu 212013, China \\ ${ }^{3}$ Industrial Technology, California State University, Fresno, CA 93740-8002, USA \\ ${ }^{4}$ College of engineering, Nanjing Agricultural University, Nanjing, Jiangsu, 210031, China
}

\section{Email address:}

qlj@ujs.edu.cn (Lijuan Qian), mjl@ujs.edu.cn (Jinlin Ma), 425534616@qq.com (Zongbo Zhang), dazhang@csufresno.edu (Daming Zhang), makaiping@njau.edu.cn (Kaiping Ma)

\section{To cite this article:}

Lijuan Qian, Jinlin Ma, Zongbo Zhang, Daming Zhang, Kaiping Ma. A Grey Relational Analysis Based Study on Green Degree Evaluation of Urban Logistics. American Journal of Applied Mathematics. Vol. 2, No. 6, 2014, pp. 209-213. doi: 10.11648/j.ajam.20140206.13

\begin{abstract}
According to the urban logistics green degree's evaluation, a weighted grey correlation analysis method based on the analytic hierarchy process is proposed to determine the weight of each index in the urban logistics green degree evaluation system, and then figure out the optimal relative degree, realizing the green degree of each urban logistics. Finally, an example was given for proving the evaluation methods' intuitive and high efficient.
\end{abstract}

Keywords: Grey Relational Analysis, Urban Logistics, Green Degree, Evaluation

\section{Introduction}

The world economy has brought serious damage to the environment. As part of the economic activity, urban logistics system is also facing environmental problems, such as polluted gas emissions, traffic congestion and improper waste disposal. Thus, countries have to develop green logistics as a key way to protect the environment and improve efficiency. Green degree of urban logistics industry has gradually become an important indicator of the standard of social development, urban construction and of living of the society. Therefore, the assessment of urban logistics green degree will not only help to identify bottlenecks in its development, but also provide theoretical guidance for the scientific development of circular economy.

\section{Determining the City Logistics Green Degree Evaluation Index System}

Green logistics refers to the reduction of environmental harm, while making the best use of logistics resources to achieve economic, social and environmental benefits. It follows four principles: 1) reduction principle, 2) reuse principle, 3) recirculation principle, 4) coexistence principles [1].
Green city logistics include green logistics operation and green logistics management [2]. Therefore, the evaluation of city logistics green degree should be considered mainly for the following indicators:

1) Intensification of resources. It's the dominant ideology of the logistics industry development. Through integrating the existing capacity resources and optimizing resource allocation and other means, logistics companies can improve resource utilization, reduce waste.

2) Green transport. Firstly, to achieve green logistics, transport routes must be optimized in order that we can shorten the transport distance, Secondly, the rational organization of the supply flow and stowage, can avoid unreasonable transport; In addition, focusing on the transport vehicle maintenance and using cleaner fuels, would be beneficial to reduce energy consumption and emissions.

3) Green warehousing. Green Warehousing needs three requirements: First, the warehouse's location should be reasonable so as to save transportation costs easily, Second, the layout of the warehouse should be scientific for realizing maximize storage volume utilization and reducing costs of inventory unit, third, the warehouse should be equipped with reasonable hardware and software facilities to improve 
management efficiency.

4) Reverse logistics. Primarily by means of reuse of waste materials, recycling and waste disposal, we could achieve minimized environmental impact [3].

\section{Multi-Level Gray Evaluation Model System}

City logistics green degree evaluation system is a gray system. Firstly, because the factors that affect the city logistics' green degree are too numerous and complex, we generally select limited key indicators to analyze while conducting the evaluation. Secondly, the link between the factors are not fully known and difficult to be quantified accurately, it is difficult to make a reasonable, accurate judgment relying solely on qualitative methods and mathematical evaluation methods in general [4]. Therefore, it is suitable for using of gray correlation analysis method combined with AHP to "whiten" the not fully ascertain relationship between the evaluation factors [5]. It can also be achieved on multiple city logistics systems to queue in line according to the quality of being friendly to the environment.

The basic idea of the evaluation: All the index value of idealized city logistics $x_{0 k}$ composing the reference sequence $X_{0}$, all measured index value of a certain city logistics $x_{i k}$ composing compared sequence $X_{i}$, we should find the correlation between $X_{0}$ and $X_{i}$. The larger the correlation, the more environmental the city logistics is. Therefore, we could judge city logistics systems based on finding the size of the correlation.

Evaluation procedure is as follows:

\subsection{To Select the Reference Sequence}

Set: $i$ is the number of the ith object (city) for evaluation, $i=1,2, \cdots, m ; k$ is the number of the $k$ th index for evaluation, $k=1,2, \cdots, n ; v_{i k}$ indicates the score of the $k$ th index for the $i$ th object. Take the ideal value $v_{0 k}$ for each indicator as entity of the reference sequence $V_{0}$, so we get,

$$
V_{0}=\left(v_{01}, v_{02}, \cdots, v_{0 n}\right)
$$

In the formula, $v_{0 k}=$ Optimum $\left(v_{i k}\right), i=1,2, \cdots, m$; $k=1,2, \cdots, n$

For a system of evaluation object of $m$, evaluation indexes of $n$, we have the following matrix:

$$
\mathrm{V}=\left(\mathrm{V}_{\mathrm{ik}}\right)_{\mathrm{m} \times \mathrm{n}}=\left[\begin{array}{cccc}
\mathrm{V}_{11} & \mathrm{~V}_{12} & \cdots & \mathrm{V}_{1 \mathrm{n}} \\
\mathrm{V}_{21} & \mathrm{~V}_{22} & \cdots & \mathrm{V}_{2 \mathrm{n}} \\
\vdots & \vdots & \ddots & \vdots \\
\mathrm{V}_{\mathrm{m} 1} & \mathrm{~V}_{\mathrm{m} 2} & \cdots & \mathrm{V}_{\mathrm{mn}}
\end{array}\right]
$$

Reference sequence to be selected is,

$$
\mathrm{V}_{0}=\left(\mathrm{v}_{01}, \mathrm{v}_{02}, \cdots, \mathrm{v}_{0 \mathrm{n}}\right)
$$

\subsection{To Normalized Index Value}

In order to compare between the various indexes, each index value needs to be normalized, normalized formula is as follows:

$$
X_{i k}=\frac{V_{i k}-\min _{i k}}{\max _{i} V_{i k}-\min _{i} V_{i k}}
$$

After the normalization process, we get:

$$
X=\left(X_{i k}\right)_{m \times n}=\left[\begin{array}{cccc}
X_{11} & X_{12} & \cdots & X_{1 n} \\
X_{21} & X_{22} & \cdots & X_{2 n} \\
\vdots & \vdots & \ddots & \vdots \\
X_{m 1} & X_{m 2} & \cdots & X_{m n}
\end{array}\right]
$$

\subsection{To Calculate the Correlation Coefficient}

Take the normalized sequence $X_{0}=\left(x_{01}, x_{02}, \cdots, x_{0 n}\right)$ as a reference sequence and $X_{i}=\left(x_{i 1}, x_{i 2}, \cdots, x_{i n}\right),(i=1,2, \cdots, m)$ as a compared sequence, Correlation coefficient is calculated as follows:

$$
\xi_{i k}=\frac{\min _{i} \min _{k}\left|X_{0 k}-X_{i k}\right|+\rho \max _{i} \max _{k}\left|X_{0 k}-X_{i k}\right|}{\left|X_{0 k}-X_{i k}\right|+\rho \max _{i} \max _{k}\left|X_{0 k}-X_{i k}\right|}
$$

Where, $i=1,2, \cdots, m ; k=1,2, \cdots, n ; \rho \in[0,1]$, which is the distinguished factor.

Calculating the correlation coefficient $\xi_{i k}(i=1,2, \cdots, m ; k=1,2, \cdots, n)$, we could obtain the correlation coefficient matrix:

$$
E=\left(\xi_{i k}\right)_{m \times n}=\left[\begin{array}{cccc}
\xi_{11} & \xi_{12} & \cdots & \xi_{1 n} \\
\xi_{21} & \xi_{22} & \cdots & \xi_{2 n} \\
\vdots & \vdots & \ddots & \vdots \\
\xi_{m 1} & \xi_{m 2} & \cdots & \xi_{m n}
\end{array}\right]
$$

Where, $\xi_{i k}$ is the correlation coefficient between $X_{i k}$ and $X_{o k}$.

\subsection{To Calculate the Single-Level Correlation}

Taking into account the importance of the indicators are not the same, the correlation calculating method of taking the weight multiplied by the correlation coefficient should be preferred. The priority weight of each index relative to the upper floor target can be calculated based on the Analytic Hierarchy Process,

$$
\mathrm{W}=\left(\mathrm{w}_{1}, \mathrm{w}_{2}, \cdots, \mathrm{w}_{\mathrm{n}}\right)
$$


In the formula, $\sum_{k=1}^{t} w_{k}=1, t$ represents the number of indicators in the layer. Correlation is calculated as follows:

$$
R=\left(r_{i}\right)_{1 \times m}=\left(r_{1}, r_{2}, \cdots, r_{m}\right)=W E^{T}
$$

\subsection{To Calculate the Ultimate Correlation of Multilayer Evaluation System}

By a multilayer evaluation system, the final correlation calculation method is as follows: Synthesize correlation coefficient of each index in $k$ layer, we could respectively obtain correlation of each index which belongs to $k-1$ layer. Then put the obtained correlation of each index in $k-1$ layer as raw data, continue to synthesize and get correlation of each index in $k-2$ layer. And so on, until we find the correlation of the highest layer so far.

\subsection{To Judge the Urban Logistics System}

According to the correlation $r_{i}(i=1,2, \cdots, m)$, judge pros and cons of each object for evaluation and sort it.

\section{Empirical Analysis}

Taking the status quo of four cities' (named City1, City2, City3, City4) logistics systems being carried out as an example to illustrate its application.

\subsection{To Establish Urban Logistics Green Degree Evaluation System}

Urban logistics green degree evaluation system is as shown in Table1. The system is composed by three lays of indicators. The first layer is called target layer, which means urban logistics green degree and is represented by $A$. The second layer is called elements layer, which consists of intensive resources $\left(B_{1}\right)$, green transport $\left(B_{2}\right)$, green warehousing $\left(B_{3}\right)$, reverse logistics $\left(B_{4}\right)$. The third layer is called index layer, which is composed of a total of 12 indicators $\left(C_{1}, C_{2}, \cdots, C_{12}\right)$.

Note: The reference values in the table refer to the maximum value of each indicator in each city logistics score.

\subsection{To Calculate the Single-Level Correlation}

Taking the status quo of City1, City2, City3, City4 $\left(V_{1}, V_{2}, V_{3}, V_{4}\right)$ four cities' logistics systems being carried out as evaluation object, organize 10 experts to rate each factor of index layer. Each evaluation scores between 0-10, the higher the score, the higher the degree of satisfaction. Using the sub-average method, we could obtain the overall score of 12 factors $v_{i k}(i=1,2,3,4 ; k=1,2,3, \cdots, 12)$ for each system and the reference scores $v_{o k}$ for the all the factors, see table 2 .

Reference sequence can be derived from Table 2,

$$
\mathrm{V}_{0}=(8,8,8,8,8,7,8,8,7,8,8,7)
$$

After each index value being normalized, take distinguished coefficient $\rho=0.5$, we will have the correlation coefficients of each indicator to each optimum value in the reference sequence,

$$
\begin{aligned}
& \xi_{1}(k)=\{1,0.67,1,0.67,0.5,1,1,0.4,0.67,0.67,0.5,0.67\} \\
& \xi_{2}(k)=\{0.5,0.67,1,0.67,0.67,0.67,0.4,0.5,1,0.5,1,1\} \\
& \xi_{3}(k)=\{0.67,1,0.5,1,1,0.67,1,0.5,1,0.5,1,1\} \\
& \xi_{4}(k)=\{0.33,0.5,0.5,1,1,0.4,0.4,1,1,1,0.4,0.67\}
\end{aligned}
$$

\subsection{Correlation Synthesis for Multilayer Structure}

Using AHP, through comparing the relative importance of every two indicators, we could get the appropriate proportion of weights to build judgment matrix for upper certain elements related elements of the underlying $[6,7,8]$. Using Yaahp software to calculate the maximum Eigen values of the judgment matrix and the corresponding eigenvectors, derived single sorting weight vector and total weight vector and get through the consistency test $[9,10]$.The results are shown in Table 3.

By the formula $R=W E^{T}$, we could calculate each city's correlation for each index of $B$ layer,

$$
R_{B_{1}}=W_{B_{1 C}} E_{B_{1 C}}^{T}=(0.78,0.613,0.89,0.443)
$$

Namely the correlation on "intensive resource" in City1, City2, City3, City4 four urban logistics system is $0.78,0.613$, 0.89 and 0.443 respectively.

Also available,

$$
\begin{aligned}
& \mathrm{R}_{\mathrm{B}_{2}}=\mathrm{W}_{\mathrm{B}_{2 \mathrm{C}}} \mathrm{E}^{\mathrm{T}}{ }_{\mathrm{B}_{2 \mathrm{C}}}=(0.7310 .697,0.849,0.759) \\
& \mathrm{R}_{\mathrm{B}_{3}}=\mathrm{W}_{\mathrm{B}_{3 \mathrm{C}}} \mathrm{E}^{\mathrm{T}}{ }_{\mathrm{B}_{3 \mathrm{C}}}=(0.851,0.550,0.939,0.611) \\
& \mathrm{R}_{\mathrm{B}_{4}}=\mathrm{W}_{\mathrm{B}_{4 \mathrm{C}}} \mathrm{E}^{\mathrm{T}}{ }_{\mathrm{B}_{4 \mathrm{C}}}=(0.595,0.806,0.806,0.678)
\end{aligned}
$$

Further, correlation of $\mathrm{A}$ at the highest level index can be obtained

$$
\begin{aligned}
& \mathrm{R}_{\mathrm{A}}=\left(\mathrm{r}_{1}, \mathrm{r}_{2}, \mathrm{r}_{3}, \mathrm{r}_{4}\right)=\mathrm{W}_{\mathrm{AB}}\left[\mathrm{R}_{\mathrm{B}_{1}}, \mathrm{R}_{\mathrm{B}_{2}}, \mathrm{R}_{\mathrm{B}_{3}}, \mathrm{R}_{\mathrm{B}_{4}}\right] \\
& =(0.748,0.659,0.872,0.609)
\end{aligned}
$$

\subsection{Comprehensive Evaluation and Sorting for the Green Degree of Urban Logistics}

In accordance with the size of the correlation available in $R_{A}$, the four urban logistics green degree integrated Sorting from low to high as follows: City $4<$ City $2<$ City $1<$ City3.

In addition, since the correlation of the city 1 on "reverse logistics" is 0.595 , and the correlation of city 2 on "green storage" is 0.55 , the correlation of city 4 on "intensive resource" is 0.443 , each correlation is at the lowest level compared with other cities in the same index which indicates it's a major bottleneck in the current development and the local government should take greater efforts to improve in the future.

\section{Conclusions}

1) Using the gray system theory evaluation of urban logistics green degree has several features of simple operation, high efficiency, less required data and clear 
explanation of the problems etc., meanwhile, it can help to expand sources of information, improve evaluation analysis of the credibility and enhance the objectivity of the evaluation results in case of incomplete information and inaccurate conditions.

2) In calculating the correlation, using AHP to determine the weight of each index, to account for differences in the degree of importance of each indicator, makes the evaluation results more reasonable and with a strong persuasiveness.

\section{Acknowledgements}

The work is supported by Jiangsu Government Scholarship for Overseas Studies and The Youth Foundation of National Natural Science Foundation of China(Grant No.71101072). The authors would also like to thank China Jiangsu University, and California University, Fresno for the support to the first author and the second author as a visiting scholar.

Table 1. Green degree evaluation index system of urban logistics

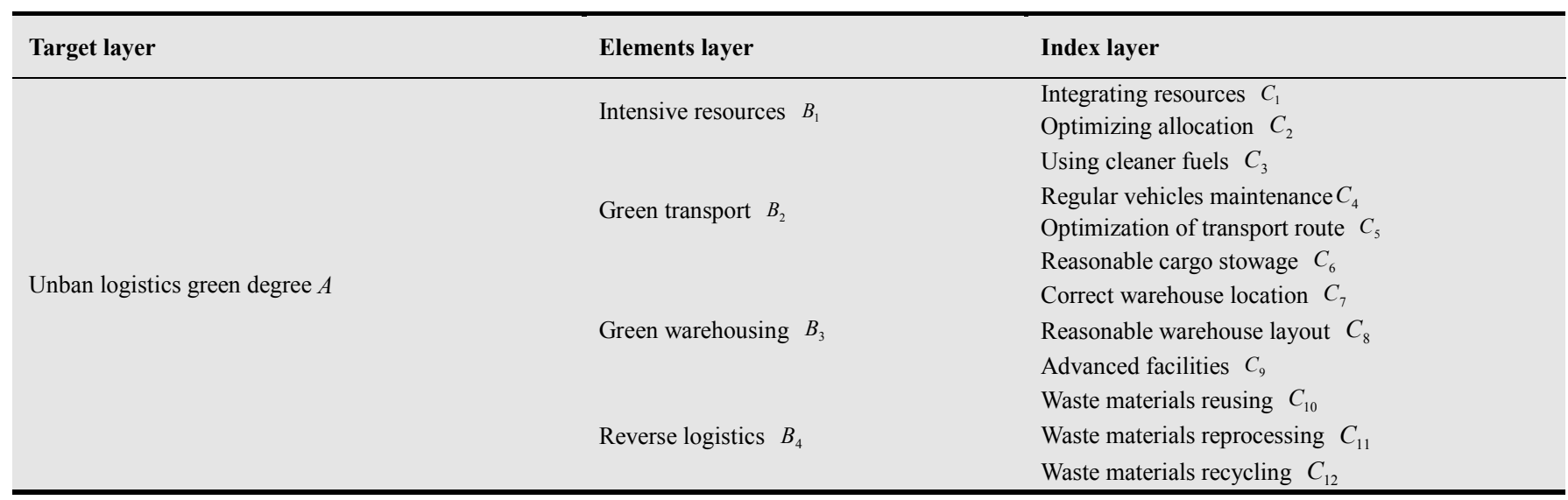

Table 2. Index scores of urban logistics and reference sequence

\begin{tabular}{|c|c|c|c|c|c|}
\hline Index layer & City1 & City2 & City3 & City4 & Reference \\
\hline \multirow{2}{*}{ Integrating resources Optimizing allocation } & 8 & 6 & 7 & 4 & 8 \\
\hline & 7 & 7 & 8 & 6 & 8 \\
\hline Using cleaner fuels & 8 & 8 & 6 & 6 & 8 \\
\hline Regular vehicles maintenance Optimization & 7 & 7 & 8 & 8 & 8 \\
\hline of transport route & 6 & 7 & 8 & 8 & 8 \\
\hline Reasonable cargo stowage & 7 & 6 & 6 & 4 & 7 \\
\hline Reasonable warehouse layout & 5 & 6 & 6 & 8 & 8 \\
\hline Advanced facilities & 6 & 7 & 7 & 7 & 7 \\
\hline Waste materials reusing & 7 & 6 & 6 & 8 & 8 \\
\hline Waste materials reprocessing Waste & 6 & 8 & 8 & 5 & 8 \\
\hline materials recycling & 6 & 7 & 7 & 6 & 7 \\
\hline
\end{tabular}

Table 3. Urban logistics green degree evaluation index weight summary

\begin{tabular}{|c|c|c|c|c|}
\hline Target layer & Elements layer & Weight & Index layer & Weight \\
\hline \multirow{4}{*}{$\begin{array}{l}\text { Unban logistics green } \\
\text { degree } A\end{array}$} & Intensive resources $B_{1}$ & 0.3618 & $\begin{array}{l}\text { Integrating resources } C_{1} \\
\text { Optimizing allocation } C_{2}\end{array}$ & $\begin{array}{l}0.3333 \\
0.6667 \\
0.129\end{array}$ \\
\hline & Green transport $B_{2}$ & 0.3270 & $\begin{array}{l}\text { Using cleaner fuels } C_{3} \\
\text { Regular vehicles maintenance } C_{4} \\
\text { Optimization of transport route } C_{5} \\
\text { Reasonable cargo stowage } C_{6}\end{array}$ & $\begin{array}{l}0.0804 \\
0.1385 \\
0.4468 \\
0.3343\end{array}$ \\
\hline & Green warehousing $B_{3}$ & 0.1635 & $\begin{array}{l}\text { Correct warehouse location } C_{7} \\
\text { Reasonable warehouse layout } C_{8} \\
\text { Advanced facilities } C_{9}\end{array}$ & $\begin{array}{l}0.6483 \\
0.1217 \\
0.2300\end{array}$ \\
\hline & Reverse logistics $B_{4}$ & 0.1477 & $\begin{array}{l}\text { Waste materials reusing } C_{10} \\
\text { Waste materials reprocessing } C_{11} \\
\text { Waste materials recycling } C_{12}\end{array}$ & $\begin{array}{l}0.3873 \\
0.4435 \\
0.1692\end{array}$ \\
\hline
\end{tabular}




\section{References}

[1] Pan Wenjun, Liang Faqi. To develop the logistics industry based on the theory of circular economy [J]. Logistics Management, 2006, 23(10): 39-43J. Clerk Maxwell, A Treatise on Electricity and Magnetism, 3rd ed., vol. 2. Oxford: Clarendon, 1892, pp.68-73.

[2] David Crawford. What the future holds for 'green logistics' [J]. Traffic Engineering and Control, 2009, 50(5): 215-217.K. Elissa, "Title of paper if known," unpublished.

[3] Hsu Hy Sonya, Alexander Christine A, Zhu Zhiwei. Understanding the reverse logistics operations of a retailer: A pilot study [J]. Industrial Management and Data Systems, 2009.109(4): 515-531.

[4] Liu Sifeng, Xie Naiming. Grey system theory and application (Fourth Edition) [M]. Beijing Science Press, 2010: 44-45.

[5] Deng Julong. Grey predication and decision[M]. Wuhan: Central institute of technology press, 1986: 56-57

[6] Du Dong, Pang Qinghua, Wu Yan. Comprehensive Evaluation of modern methods and case selection [M].Beijing: Tsinghua University Press, 2005: 14-20.

[7] Aya Zeki, Izadbakhsh HR, A combined fuzzy AHP-goal programming approach to assembly-line selection [J]. Journal of Intelligent and Fuzzy Systems, 2007, 18(4): 345-362.

[8] Coelli TJ, Prasada Rao DS, O’Donnell CJ, et al. Introduction to efficiency and productivity analysis $[\mathrm{M}]$. Second Edition. New York: Springer Press, 2005: 202-230.

[9] Andersen B, Fagerhaug T. Performance measurement of logistics processes [J]. Journal of Operation Management, 2003(20): 19-32.

[10] Liu Ying Ming, Luo Mao Kang. Fuzzy topology [M]. Singapore: World Scientific, 1997: 120-131. 